\title{
On the set of fixed points of automorphisms of bordered Klein surfaces
}

\author{
José Manuel Gamboa and Grzegorz Gromadzki
}

\begin{abstract}
The nature of the set of points fixed by automorphisms of Riemann or unbordered nonorientable Klein surfaces as well as quantitative formulae for them were found by Macbeath, Izquierdo, Singerman and Gromadzki in a series of papers. The possible set of points fixed by involutions of bordered Klein surfaces has been found by Bujalance, Costa, Natanzon and Singerman who showed that it consists of isolated fixed points, ovals and chains of arcs. They classified involutions of such surfaces, up to topological conjugacy in these terms. Here we give formulae for the number of elements of each type, also for non-involutory automorphisms, in terms of the topological type of the action of the group of dianalytic automorphisms. Finally we give some illustrative examples concerning bordered Klein surfaces with large groups of automorphisms already considered by May and Bujalance.
\end{abstract}

\section{Introduction}

Macbeath found in [11] a formula for the number of points fixed by an arbitrary analytic automorphism $\varphi$ of a compact Riemann surface $X$ in terms of the topological type of the group $G$ of conformal automorphisms of $X$, which corresponds to a so called smooth epimorphism $\theta: \Lambda \rightarrow G$. Later, Izquierdo and Singerman [9] obtained in such terms formulae for $G$ a cyclic group of automorphisms of an unbordered non-orientable compact Klein surface, and Gromadzki [8] provided such formulae for arbitrary group $G$ of all dianalytic automorphisms of $X$. Here we deal with the case in which $X$ is a compact bordered Klein surface. Then the group $\Lambda$ above (which is a Fuchsian group in the case of Riemann surfaces and analytic automorphisms) is an NEC group, that is, a discrete subgroup of the group of isometries of the hyperbolic plane including orientation reversing ones which uniformises the compact quotient surface $X / G$.

Mathematics Subject Classification (2010): Primary 30F10; Secondary 14H55.

Keywords: Automorphisms of Riemann (Klein) surfaces, symmetries of Riemann surfaces, fixed points, Fuchsian, NEC groups. 
The set of points fixed by an automorphism $\varphi$ of a classical Riemann surface $X$ consists either of a finite number of isolated points if $\varphi$ is analytic or of a finite number of ovals if $\varphi$ is an antianalytic involution. In particular, Harnack's Theorem (see e.g. [3] for modern exposition), which is considered as the first relevant result in Real Algebraic Geometry, states that the number of such ovals is bounded above by $g+1$, where $g$ is the genus of $X$. If we allow $X$ to be non-orientable then both types of points fixed by a dianalytic automorphism can simultaneously occur.

In this paper $X$ is a compact bordered Klein surface, which in particular has a finite number of connected components. By the well-known categorial coequivalence, see e.g. [5], the problem we deal with is equivalent to study the set of fixed points of birational automorphisms of real irreducible algebraic curves. Let

$$
\operatorname{Fix}(X)=\bigcup_{\operatorname{id} \neq \varphi \in \operatorname{Aut}(X)} \operatorname{Fix}(\varphi)
$$

The first, and actually the only important work in this direction is [4], where the authors described the nature of the set $\operatorname{Fix}(\sigma)$ of points fixed by an involution $\sigma$, classified involutions up to topological conjugacy by means of certain numerical invariants concerning $\operatorname{Fix}(\sigma)$ and showed that it is composed by a finite number of isolated fixed points, a finite number of ovals, which can be divided into twisted and non-twisted, and a finite number of chains of closed $\operatorname{arcs}$ on $X$, also divided into twisted and non-twisted.

Here we show, in addition, that the set of points fixed by an automorphism which is not an involution consists of two parts:

(i) a finite set of points isolated in $\operatorname{Fix}(X)$,

(ii) a finite set of points not being isolated in $\operatorname{Fix}(X)$, i.e. lying on the intersections of two ovals, two arcs or an arc and an oval.

Moreover, we find in Theorems 3.2, 3.3 and 3.4 formulae for the number of isolated fixed points of each type and the number of ovals and arcs for an arbitrary automorphism $\varphi \in G=\operatorname{Aut}(X)$ just in terms of $G$ and the topological type of the action of $G$ on the surface $X$. Moreover, we supply in Section 4 some illustrative examples concerning the set of fixed points of bordered Klein surfaces with large groups of automorphisms already considered by May and Bujalance.

\section{Preliminaries}

We shall use combinatorial methods based on the Riemann uniformization theorem and the combinatorial theory of Fuchsian and NEC groups as in [5], where the reader can find all necessary notions and facts.

By a Klein surface we mean a compact topological surface with a dianalytic structure, which roughly speaking differs from the classical conformal structure by the fact that reflections $z \mapsto-\bar{z}$ are allowed as transition functions of charts, see [1] for details. Given such a surface $X$, there is a double Riemann covering $\tilde{X}$ together 
with an antianalytic involution $\tau$, known as the structural symmetry, such that the orbit space $\widetilde{X} / \tau$ with the natural structure of Klein surface inherited from $\tilde{X}$ is dianalytically isomorphic to $X$, see [1]. The surface $\widetilde{X}$ is called the complex double of $X$. This makes the study of automorphisms of Klein surfaces equivalent to the study of dianalytic automorphisms of $\widetilde{X}$ centralizing $\tau$. We shall use this approach here in some proofs. The algebraic genus of $X$ is defined to be the topological genus of $\widetilde{X}$. Now, the Riemann uniformization theorem for Riemann surfaces and the above fact yield the equivalent theorem for Klein surfaces: each Klein surface $X$ can be represented as the orbit space $\mathcal{H} / \Gamma$ of the hyperbolic plane $\mathcal{H}$ with respect to the action of a surface non-euclidean crystallographic group (NECgroup in short), and its group of dianalytic automorphisms can be represented as the factor group $\Lambda / \Gamma$ for some larger NEC-group $\Lambda$.

An NEC-group $\Lambda$ is a (finitely generated) discrete subgroup of the group $\mathcal{G}$ of hyperbolic isometries, including those which reverse orientation, of the hyperbolic plane $\mathcal{H}$ with compact orbit space. If reflections are the only torsion elements in $\Lambda$, then this group is called a surface NEC-group. Macbeath [10] and Wilkie [15] associated to every NEC-group $\Lambda$ a signature, which determines its algebraic structure. It has the form

$$
\left(g ; \pm ;\left[m_{1}, \ldots, m_{r}\right] ;\left\{\left(n_{11}, \ldots, n_{1 s_{1}}\right), \ldots,\left(n_{k 1}, \ldots, n_{k s_{k}}\right)\right\}\right) .
$$

A surface NEC group $\Gamma$ has signature $(g ; \pm ;[-] ;\{(-), . . . .,(-)\})$ and the algebraic genus of $\mathcal{H} / \Gamma$ equals $\varepsilon g+k-1$, where $\varepsilon=2$ if the sign is + and $\varepsilon=1$ if the sign is - . The numbers $m_{i} \geq 2$ are called the proper periods, the sequences $C_{i}=\left(n_{i 1}, \ldots, n_{i s_{i}}\right)$ the period cycles, the numbers $n_{i j} \geq 2$ the link periods, $s_{i}$ is the length of the period cycle $C_{i}$ and $g \geq 0$ is said to be the orbit genus of $\Lambda$.

The orbit space $\mathcal{H} / \Lambda$ is a surface having $k$ boundary components, orientable or not according to the sign being + or - and having topological genus $g$.

A group $\Lambda$ with signature (2.1) has a presentation with the following generators:

$$
x_{1}, \ldots, x_{r}, e_{i}, c_{i j}, \quad 1 \leq i \leq k, 0 \leq j \leq s_{i}
$$

and

$$
a_{1}, b_{1}, \ldots, a_{g}, b_{g} \text { if the sign is }+, \text { or } d_{1}, \ldots, d_{g} \text { otherwise, }
$$

and relators:

$$
x_{i}^{m_{i}}, \quad i=1, \ldots, r, \quad c_{i j}^{2},\left(c_{i j-1} c_{i j}\right)^{n_{i j}}, c_{i 0} e_{i}^{-1} c_{i s_{i}} e_{i}, \quad i=1, \ldots, k, j=0, \ldots, s_{i}
$$

and

$$
x_{1} \cdots x_{r} e_{1} \cdots e_{k} a_{1} b_{1} a_{1}^{-1} b_{1}^{-1} \cdots a_{g} b_{g} a_{g}^{-1} b_{g}^{-1} \quad \text { or } \quad x_{1} \cdots x_{r} e_{1} \cdots e_{k} d_{1}^{2} \cdots d_{g}^{2},
$$

according to whether the sign is + or - . The elements $x_{i}$ are elliptic transformations associated to the proper period $m_{i}$, and $c_{i j}$ are reflections associated to the period cycle $C_{i}$. Every element of finite order in $\Lambda$ is conjugate either to a generating reflection $c_{i j}$, to a power of some elliptic generator $x_{i}$ or to a power of the product of two consecutive generating reflections $c_{i j-1}, c_{i j}$. Any system of generators of an NEC-group satisfying the above relations will be called a canonical system of generators and its elements will be called canonical generators. 
Every NEC-group $\Lambda$ has an associated fundamental region whose hyperbolic area $\mu(\Lambda)$ is given by

$$
\mu(\Lambda)=2 \pi\left(\varepsilon g+k-2+\sum_{i=1}^{r}\left(1-\frac{1}{m_{i}}\right)+\frac{1}{2} \sum_{i=1}^{k} \sum_{i=1}^{s_{i}}\left(1-\frac{1}{n_{i j}}\right)\right),
$$

where $\varepsilon$ is defined as above. It is known that an abstract group with the presentation given by the above canonical generators and relations can be realized as an NEC-group with the signature in (2.1) if and only if the right hand side of (2.2) is positive. If $\Lambda^{\prime}$ is a subgroup of finite index in an NEC-group $\Lambda$ then it is an NEC-group itself. Moreover, the Hurwitz-Riemann formula applied to the covering $\mathcal{H} / \Lambda^{\prime} \rightarrow \mathcal{H} / \Lambda$ reads

$$
\mu(\Lambda)\left[\Lambda: \Lambda^{\prime}\right]=\mu\left(\Lambda^{\prime}\right)
$$

Any dianalytic action of a finite group $G$ on a bordered Klein surface of algebraic genus $p$ is determined by an epimorphism $\theta: \Lambda \rightarrow G$, where $\Lambda$ is an NEC-group, $\theta$ preserves the orders of canonical elliptic generators and the orders of products of consecutive canonical reflections, and $\mu(\Lambda)=2 \pi|G|(p-1)$. Such an epimorphism is said to be smooth, and two such epimorphisms $\theta: \Lambda \rightarrow G$ and $\theta^{\prime}: \Lambda^{\prime} \rightarrow G^{\prime}$ are said to define topologically equivalent actions if the diagram

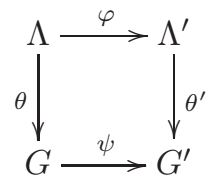

commute for some isomorphisms $\varphi: \Lambda \rightarrow \Lambda^{\prime}$ and $\psi: G \rightarrow G^{\prime}$. It is worthwhile mentioning that all the results obtained in this paper are invariant under this relation; therefore we will freely substitute $(\theta, \Lambda, G)$ by $\left(\theta^{\prime}, \Lambda^{\prime}, G^{\prime}\right)$ if necessary.

Throughout the article, $\mathrm{Z}_{n}$ and $\mathrm{D}_{n}$ denote the cyclic and the dihedral group of order $n$ and $2 n$, respectively; $\mathrm{C}(G, \varphi)$ and $\mathrm{N}_{G}(\langle\varphi\rangle)$ denote, respectively, the centralizer of $\varphi$ in $G$ and the normalizer of the subgroup generated by $\varphi$ in $G$.

\section{Quantitative aspects of the set of fixed points}

All throughout the remainder of the paper, given a bordered Klein surface $X$ we will denote by $\pi: \mathcal{H} \rightarrow X=\mathcal{H} / \Gamma$ the canonical projection, and we fix an automorphism $\varphi$ of $X$ (that we will denote $\sigma$ if it is involutory), and a dianalytic action of the group $G=\operatorname{Aut}(X)$ on $X$ that is given by a smooth epimorphism $\theta: \Lambda \rightarrow G$.

First, we classify the fixed points of a non-involutory automorphism of a bordered Klein surface $X$ into two types, according to whether they are isolated in $\operatorname{Fix}(X)$ or not. 
Lemma 3.1. The set of points fixed by a non-involutory automorphism of a bordered Klein surface $X$ consists of two subsets: a finite set of points isolated in $\operatorname{Fix}(X)$ and a finite set of points lying on the intersections of either two ovals or two arcs or an arc and an oval.

Proof. Consider the universal covering $\pi: \mathcal{H} \rightarrow X$ and, given a non-involutory automorphism $\varphi \in G$, let $\lambda \in \Lambda$ with $\theta(\lambda)=\varphi$. Consider the commutative diagram

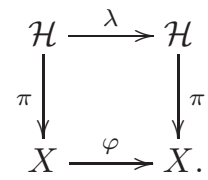

Hence, for each point $x=\pi(h) \in X$, we have $\varphi(x)=\pi(\lambda(h))$. Thus $x=\pi(h)$ is fixed by $\varphi$ if and only if $\lambda(h)=\gamma(h)$ for some $\gamma \in \Gamma$, which means that $\gamma^{-1} \lambda$ fixes $h$. Therefore $\gamma^{-1} \lambda$ has finite order and, indeed, it is an elliptic transformation because its order is different to 2 since $\theta\left(\gamma^{-1} \lambda\right)=\varphi$ is not an involution. Now, if $\gamma^{-1} \lambda$ is not a product of two reflections, then $x=\pi(h)$ is isolated in $\operatorname{Fix}(X)$; otherwise it lies on the intersection of either two ovals, or two arcs, or an arc and an oval.

We shall refer to the fixed points occurring in the above lemma as to fixed points of the first and the second type respectively. We enumerate in the next theorem the number of isolated fixed points of both types for each automorphism of a bordered Klein surface.

Theorem 3.2. Let $\varphi$ be an automorphism of a bordered Klein surface. Then, the number of isolated fixed points of $\varphi$ of the first and the second type is given by

$$
\left|\mathrm{N}_{G}(\langle\varphi\rangle)\right|\left(\sum 1 / m_{i}\right) \text { and }\left|\mathrm{N}_{G}(\langle\varphi\rangle)\right|\left(\sum 1 / n_{i j}\right)
$$

respectively, where the sums run over the canonical elliptic generators and the products of consecutive canonical reflections for which $\varphi$ is conjugate to either a power of $\theta\left(x_{i}\right)$ or $\theta\left(c_{i j-1} c_{i j}\right)$, whenever $\theta\left(c_{i j-1}\right) \neq 1 \neq \theta\left(c_{i j}\right)$.

Proof. If $\varphi$ has order $n$, then the number of points fixed by $\varphi$ equals the number of conjugacy classes of cyclic subgroups of order $n$ of the group $\Gamma_{\varphi}=\theta^{-1}(\langle\varphi\rangle)$. Clearly, each of such subgroups is generated by an elliptic element. But each elliptic element is conjugate either to a power of some canonical elliptic generator $x_{i}$ of $\Lambda$ or to a power of the product $c_{i j-1} c_{i j}$ of two consecutive canonical reflections of $\Lambda$; by abuse of language we say that the fixed points of $\varphi$ are produced by $x_{i}$ or by $c_{i j-1} c_{i j}$ in each one of these cases, respectively.

Hence, we have to find how many points fixed by $\varphi$ are produced by each element $x_{i}$ and by each product $c_{i j-1} c_{i j}$, which in turn will provide us, after addition, the number of fixed points of $\varphi$ of the first and the second type.

Assume first that $x_{i}$ produces fixed points of $\varphi$. Then, since conjugate elements have the same number of fixed points, after exchanging $\varphi$ by a suitable conjugate we may assume that $x_{i}^{n_{i}} \in \Gamma_{\varphi}$ for $n_{i}=m_{i} / n$. 
Now, $w x_{i}^{n_{i}} w^{-1} \in \Gamma_{\varphi}$ if and only if $w \in \theta^{-1}\left(\mathrm{~N}_{G}(\langle\varphi\rangle)\right)$. Observe, however, that $w x_{i}^{n_{i}} w^{-1}$ and $v x_{i}^{n_{i}} v^{-1}$ are conjugate in $\Gamma_{\varphi}$ if and only if $w^{-1} \gamma v \in \mathrm{N}_{\Lambda}\left(\left\langle x_{i}^{n_{i}}\right\rangle\right)=\left\langle x_{i}\right\rangle$ for some $\gamma \in \Gamma_{\varphi}$, which means that $w^{-1} v \in\left\langle x_{i}\right\rangle \Gamma_{\varphi}$. Thus, the number of fixed points of $\varphi$ produced by $x_{i}$ equals

$$
\left[\theta^{-1}\left(\mathrm{~N}_{G}(\langle\varphi\rangle)\right):\left\langle x_{i}\right\rangle \Gamma_{\varphi}\right]=\left[\theta^{-1}\left(\mathrm{~N}_{G}(\langle\varphi\rangle)\right) / \Gamma:\left\langle x_{i}\right\rangle \Gamma_{\varphi} / \Gamma\right]=\left|\mathrm{N}_{G}(\langle\varphi\rangle)\right| / m_{i}
$$

This proves the first part of the statement. The argument to prove the second part is rather similar.

Indeed, assume that $c_{i j-1} c_{i j}$ produces fixed points of $\varphi$. Then, we may assume that $\left(c_{i j-1} c_{i j}\right)^{m_{i j}} \in \Gamma_{\varphi}$ for $m_{i j}=n_{i j} / n$. Furthermore, $w\left(c_{i j-1} c_{i j}\right)^{m_{i j}} w^{-1} \in \Gamma_{\varphi}$ if and only if $w \in \theta^{-1}\left(\mathrm{~N}_{G}(\langle\varphi\rangle)\right)$. On the other hand $w\left(c_{i j-1} c_{i j}\right)^{m_{i j}} w^{-1}$ and $v\left(c_{i j-1} c_{i j}\right)^{m_{i j}} v^{-1}$ are conjugate in $\Gamma_{\varphi}$ if and only if $w^{-1} \gamma v$ belongs to

$$
\mathrm{N}_{\Lambda}\left(\left\langle\left(c_{i j-1} c_{i j}\right)^{m_{i j}}\right\rangle\right)=\left\langle c_{i j-1} c_{i j}\right\rangle
$$

for some $\gamma \in \Gamma_{\varphi}$, which means that $w^{-1} v \in\left\langle c_{i j-1} c_{i j}\right\rangle \Gamma_{\varphi}$. Hence $c_{i j-1} c_{i j}$ produces

$$
\begin{aligned}
{\left[\theta^{-1}\left(\mathrm{~N}_{G}(\langle\varphi\rangle)\right):\left\langle c_{i j-1} c_{i j}\right\rangle \Gamma_{\varphi}\right] } & =\left[\theta\left(\theta^{-1}\left(\mathrm{~N}_{G}(\langle\varphi\rangle)\right)\right): \theta\left(\left\langle c_{i j-1} c_{i j}\right\rangle \Gamma_{\varphi}\right)\right] \\
& =\left[\mathrm{N}_{G}(\langle\varphi\rangle):\left\langle\theta\left(c_{i j-1} c_{i j}\right)\right\rangle\right] \\
& =\left|\mathrm{N}_{G}(\langle\varphi\rangle)\right| / n_{i j}
\end{aligned}
$$

fixed points of $\varphi$, and this proves the second part of the statement.

Our next result concerns the enumeration of the number of ovals of the set of fixed points of an involution on a bordered Klein surface. An oval on $X$ is a simple closed curve on the interior of $X$ consisting of fixed points of some involution.

Theorem 3.3. The number of ovals of the set of fixed points of an involution $\sigma$ of a bordered Klein surface is given by

$$
\sum[\mathrm{C}(\theta(\Lambda), \theta(c)): \theta(\mathrm{C}(\Lambda, c))]
$$

where the sum runs over all non-conjugate canonical reflections $c \in \Lambda$ whose images by $\theta$ are conjugate in $G$ to $\sigma$ and correspond, either to empty period cycles of the signature of $\Lambda$ or to nonempty period cycles for which those neighbouring canonical reflections to $c$ are mapped by $\theta$ to nontrivial elements of $G$.

Proof. We have to count how many reflections of $\Lambda$ belong to the subgroup $\Gamma_{\sigma}=$ $\theta^{-1}(\langle\sigma\rangle)$ and are pairwise non-conjugate in $\Gamma_{\sigma}$. If $\operatorname{Fix}(\sigma)$ contains an oval, then $\sigma$ is conjugate to $\theta\left(c_{i}\right)$ for some canonical reflection $c_{i}$ of $\Lambda$. In fact we may assume, without loss of generality, that $\theta\left(c_{i}\right)=\sigma$, because conjugate symmetries have the same number of ovals. Observe that for $w \in \Lambda$, the conjugate $c_{i}^{w}$ belongs to $\Gamma_{\sigma}$ if and only if $w \in \theta^{-1}\left(\mathrm{C}\left(G, \theta\left(c_{i}\right)\right)\right)$. Denote the last group by $\mathrm{C}_{i}$ and observe that it normalizes $\Gamma_{\sigma}$. Thus for $v, w \in \mathrm{C}_{i}$, the reflections $c_{i}^{v}$ and $c_{i}^{w}$ of $\Gamma_{\sigma}$ are conjugate in $\Gamma_{\sigma}$ if and only if $w^{-1} v \in \mathrm{C}\left(\Lambda, c_{i}\right) \Gamma_{\sigma}$. Consequently, conjugates of $c_{i}$ give rise to

$$
\left[\mathrm{C}_{i}: \mathrm{C}\left(\Lambda, c_{i}\right) \Gamma_{\sigma}\right]=\left[\mathrm{C}\left(G, \theta\left(c_{i}\right)\right): \theta\left(\mathrm{C}\left(\Lambda, c_{i}\right)\right)\right]
$$


empty period cycles in $\Gamma_{\sigma}$. Let now $c_{\ell}^{w} \in \Gamma_{\sigma}$ for some $\ell \neq i$ and $w \in \Lambda$. Then $\theta(w) \theta\left(c_{\ell}\right) \theta(w)^{-1}=\sigma$ and so

$$
w \mathrm{C}_{\ell} w^{-1}=\mathrm{C}_{i} .
$$

Indeed, if $\lambda \in w \mathrm{C}_{\ell} w^{-1}$, then $\theta(w)^{-1} \theta(\lambda) \theta(w) \in \mathrm{C}\left(G, \theta\left(c_{\ell}\right)\right)$. Thus $\theta(\lambda)$ centralizes $\sigma$ and so $\lambda \in \mathrm{C}_{i}$. Conversely, if $\lambda \in \mathrm{C}_{i}$, then $\theta(\lambda)$ normalizes $\sigma$. Hence $\theta(\lambda) \theta(w) \theta\left(c_{\ell}\right) \theta(w)^{-1} \theta(\lambda)^{-1}=\sigma$, which in turn means that $\theta(w)^{-1} \theta(\lambda) \theta(w)$ centralizes $\theta\left(c_{\ell}\right)$, and so $\lambda \in w \mathrm{C}_{\ell} w^{-1}$, proving (3.1).

Furthermore, $c_{\ell}^{v} \in \Gamma_{\sigma}$ if and only if $v w^{-1} \in \mathrm{C}_{i}$. Indeed, if this is the case, then $\theta(v) \theta\left(c_{\ell}\right) \theta(v)^{-1}=\sigma$ and so $\theta(w)^{-1} \theta(v) \in \mathrm{C}\left(\Lambda, \theta\left(c_{\ell}\right)\right)$. Thus $w^{-1} v \in \mathrm{C}_{\ell}$, which implies that $v w^{-1} \in w \mathrm{C}_{\ell} w^{-1}=\mathrm{C}_{i}$. The converse is similar and we omit its proof.

Finally, given $u, u^{\prime} \in \mathrm{C}_{i}$ and the products $v=u w, v^{\prime}=u^{\prime} w$, the reflections $c_{\ell}^{v}$ and $c_{\ell}^{v^{\prime}}$ are conjugate in the subgroup $\Gamma_{\sigma}$ if and only if $v^{-1} v^{\prime} \in \mathrm{C}\left(\Lambda, c_{\ell}\right) w^{-1} \Gamma_{\sigma} w=$ $\mathrm{C}\left(\Lambda, c_{\ell}\right) \Gamma$, which means that $u^{-1} u^{\prime} \in w \mathrm{C}\left(\Lambda, c_{\ell}\right) \Gamma w^{-1}$. Therefore, by (3.1), the conjugates of $c_{\ell}$ give rise to

$$
\left[\mathrm{C}_{i}: w \mathrm{C}\left(\Lambda, c_{\ell}\right) \Gamma w^{-1}\right]=\left[\mathrm{C}_{\ell}: \mathrm{C}\left(\Lambda, c_{\ell}\right) \Gamma\right]=\left[\mathrm{C}\left(\theta(\Lambda), \theta\left(c_{\ell}\right)\right): \theta\left(\mathrm{C}\left(\Lambda, c_{\ell}\right)\right)\right]
$$

empty period cycles in $\Gamma_{\sigma}$, and so the result follows.

To finish this section we count the number of arcs in the set of fixed points of an involution on a bordered Klein surface. An $\operatorname{arc}$ on $X$ is a simple curve, homeomorphic to a closed interval, which meets either one or two boundary components of $X$ at its endpoints.

Theorem 3.4. The set of fixed points of an involution $\sigma$ on a bordered Klein surface $X$ contains

$$
\sum[\mathrm{C}(\theta(\Lambda), \theta(c)): \theta(\mathrm{C}(\Lambda, c))]
$$

arcs, where the sum is taken over all non-conjugate canonical reflections in $\Lambda$ whose images by $\theta$ are conjugate in $G$ to $\sigma$, in such a way that at least one neighbouring canonical reflection to $c$ is mapped by $\theta$ into the identity element.

Proof. Let us write $X=\mathcal{H} / \Gamma$ and $G=\Lambda / \Gamma$, where $\Gamma$ is a surface NEC group. Consider the commutative diagrams
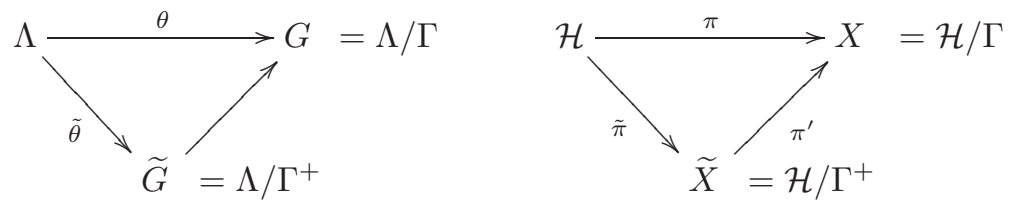

where $\Gamma^{+}$is the subgroup of $\Gamma$ consisting of all its orientation preserving elements, $\widetilde{X}$ is the canonical Riemann double cover of $X$ and $\widetilde{G}$ is a group of automorphisms of $\widetilde{X}$. Let $\tau$ be the structural symmetry of $\widetilde{X}$.

The set $\operatorname{Fix}(\sigma)$ of fixed points of $\sigma$ being non-discrete, there exists a reflection of $\Lambda$ whose image by $\theta$ is $\sigma$. Let also denote by $\sigma$, for the sake of notational 
convenience, its lifting to $\widetilde{X}$, which is also a symmetry. Clearly, the cardinality of $\operatorname{Fix}(\sigma) \cap \operatorname{Fix}(\tau)$ equals $2 N$, where $N$ is the number of arcs of $\sigma$ we are looking for. Now an oval of $\sigma$ in $\widetilde{X}$ corresponds to a conjugate element $w c w^{-1}$ of some canonical reflection $c$. But either such an oval meets twice a unique oval of $\tau$ or it meets two distinct ovals of $\tau$, once each one, according as the number of canonical reflections neighbouring to $c$ mapped by $\widetilde{\theta}$ into $\tau$ equals one or two. Let $c^{\prime}$ and $\left\{c^{\prime}, c^{\prime \prime}\right\}$ denote, respectively, the canonical reflections neighbouring to $c$ mapped by $\tilde{\theta}$ into $\tau$. Observe that every conjugate element of these reflections $c^{\prime}$ and $c^{\prime \prime}$ is also mapped into $\tau$ because $\tau$ is a central element of $\widetilde{G}$. But, by the proof of Theorem 3.3, such a reflection $c$ produces $[\mathrm{C}(\widetilde{G}, \sigma): \widetilde{\theta}(\mathrm{C}(\Lambda, c))]$ ovals of $\sigma$. Using again the centrality of $\tau$, we see that the last number equals $[\mathrm{C}(G, \sigma): \theta(\mathrm{C}(\Lambda, c))]=$ $[\mathrm{C}(\theta(\Lambda), \theta(c)): \theta(\mathrm{C}(\Lambda, c))]$, and so the theorem follows.

Remark 3.5. To apply these theorems to compute the number of ovals and arcs fixed by an involution $\sigma$ of a bordered Klein surface it is necessary to calculate the indices $[\mathrm{C}(\theta(\Lambda), \theta(c)): \theta(\mathrm{C}(\Lambda, c))]$ for the canonical reflections $c \in \Lambda$ mapped by $\theta$ into conjugates of $\sigma$. The algebraic type of these centralizers was found by Singerman in his thesis [13] (see also [14]). However, what makes Theorems 3.3 and 3.4 effective, is the fact that, by going a bit more into details in Singerman's papers, one can find explicit generators for these groups (e.g., [3] and [7]).

\section{Examples: Bujalance-May surfaces}

It is well known that the group of automorphisms of a bordered Klein surface of algebraic genus $p \geq 2$ has at most $12(p-1)$ elements, and May proved in [12] that given $p \geq 2$ there exists a bordered Klein surface of algebraic genus $p$ whose group of automorphisms has, at least, $4(p+\mu)$ elements, with $\mu=1$ if the surface is orientable and $\mu=0$ otherwise. Furthermore there are infinitely many values of $p$ for which there is no Klein surface of such algebraic genus with a bigger group of automorphisms, and Bujalance described in [2] both the topological type and the groups of automorphisms of such extremal surfaces. Here we shall find the topological type of the set of fixed points for these surfaces, which we call Bujalance-May surfaces.

Example 4.1. Orientable Bujalance-May surfaces. Let $p \geq 2$ with $p \neq$ $5,11,29$, and let $X=\mathcal{H} / \Gamma$ be a bordered Klein surface of algebraic genus $p$ whose automorphism group $G$ has order $4(p+1)$. Bujalance proved in Theorem 2.1 of [2] that in this situation the group $G=\operatorname{Aut}(X)=\Lambda / \Gamma$ is the semidirect product

$$
G=\mathrm{D}_{p+1} \rtimes \mathrm{Z}_{2}=\left\langle x, y, z: x^{2}, y^{2}, z^{2},(x y)^{p+1},(y z)^{2}, z x z(y x)^{r} y\right\rangle
$$

for some $r \in \mathbb{Z}$ such that $r^{2} \equiv 1 \bmod (p+1)$, and the signature of $\Lambda$ is

$$
(0,+,[-],\{(p+1,2,2,2)\}) .
$$

The corresponding canonical epimorphism $\theta: \Lambda \rightarrow G$ is induced by the assignment

$$
\theta(e)=1, \quad \theta\left(c_{0}\right)=\theta\left(c_{4}\right)=y, \quad \theta\left(c_{1}\right)=x, \quad \theta\left(c_{2}\right)=1, \quad \theta\left(c_{3}\right)=z .
$$


Using the formulae in Theorems 3.2, 3.3 and 3.4, we obtain, for $p \equiv 1 \bmod (2)$,

\begin{tabular}{|c|c|c|c|c|}
\hline $\begin{array}{c}\text { Representative } \\
\text { of a conjugacy } \\
\text { class }\end{array}$ & $\begin{array}{c}\text { Isolated fixed } \\
\text { points of the } \\
\text { first type }\end{array}$ & $\begin{array}{c}\text { Isolated fixed } \\
\text { points of the } \\
\text { second type }\end{array}$ & Ovals & Arcs \\
\hline$x$ & 0 & 0 & 0 & 2 \\
$y$ & 0 & 0 & 1 & 0 \\
$z$ & 0 & 0 & 0 & $(r+1, p+1)$ \\
$x y$ & 0 & 4 & 0 & 0 \\
$y z$ & 0 & $2(r-1, p+1)$ & 0 & 0 \\
\hline
\end{tabular}

where $(r-1, p+1)$ is the greatest common divisor of $r-1$ and $p+1$ and, by convention, $(r-1, p+1)=p+1$ if $r=1$.

Concerning the case of even $p$, observe that the involutions $x$ and $y$ are conjugate. Indeed, let $j=(p+2) / 2$ and $\alpha=(x y)^{j} y \in G$. Then,

$$
\begin{aligned}
\alpha y \alpha^{-1} & =(x y)^{j} y y y(x y)^{-j}=(x y)^{j} y(x y)^{-j}=(x y)^{j} y(x y)^{j}(x y)^{-2 j} \\
& =y(y x)^{2 j}=(x y)^{2 j-1} x=(x y)^{p+1} x=x .
\end{aligned}
$$

A straightforward computation shows that the corresponding results for $p \equiv 0$ mod (2) are the following:

\begin{tabular}{|c|c|c|c|c|}
\hline $\begin{array}{c}\text { Representative } \\
\text { of a conjugacy } \\
\text { class }\end{array}$ & $\begin{array}{c}\text { Isolated fixed } \\
\text { points of the } \\
\text { first type }\end{array}$ & $\begin{array}{c}\text { Isolated fixed } \\
\text { points of the } \\
\text { second type }\end{array}$ & Ovals & Arcs \\
\hline$y$ & 0 & 0 & 1 & 1 \\
$z$ & 0 & 0 & 0 & $(r+1, p+1)$ \\
$x y$ & 0 & 4 & 0 & 0 \\
$y z$ & 0 & $2(r-1, p+1)$ & 0 & 0 \\
\hline
\end{tabular}

All entries in the tables above are easily calculated by applying Theorems 3.2 , 3.3 and 3.4. To show the kind of arguments used in the computations, just consider, by way of illustration, how to see that the set $\operatorname{Fix}(z)$ of fixed points of the involution $z$ contains $(r+1, p+1)$ arcs.

Note that $c_{2}$ is the only canonical reflection of $\Lambda$ which is mapped by $\theta$ into the unit. Since $\theta\left(c_{1}\right)=x$ and $\theta\left(c_{3}\right)=z$, the sets of fixed points $\operatorname{Fix}(x)$ and $\operatorname{Fix}(z)$ are the only ones containing arcs. Moreover, $x$ and $z$ are not conjugate in $G$. Suppose, by way of contradiction, that $z=\xi x \xi^{-1}$ for some $\xi=(x y)^{i} y^{j} z^{k} \in G$, with $0 \leq i \leq p$ and $0 \leq j, k \leq 1$. Thus $k=1$, because $z \notin\langle x, y\rangle$, that is,

$$
z=(x y)^{i} y^{j} z x z y^{j}(x y)^{-i}=(x y)^{i} y^{j}(y x)^{r} y z z y^{j}(x y)^{-i} \in\langle x, y\rangle,
$$

an absurd. Therefore, by Theorem 3.4, it follows that Fix $(z)$ contains

$$
\left[\mathrm{C}(G, z): \theta\left(\mathrm{C}\left(\Lambda, c_{3}\right)\right)\right]=\frac{|\mathrm{C}(G, z)|}{\left|\theta\left(\mathrm{C}\left(\Lambda, c_{3}\right)\right)\right|}
$$


arcs. To compute $|\mathrm{C}(G, z)|$ observe that $z$ commutes with all elements of the subgroup $\langle y, z\rangle$, and so $(x y)^{i} y^{j} z^{k} \in \mathrm{C}(G, z)$ if and only if $(x y)^{i} \in \mathrm{C}(G, z)$. This way $|\mathrm{C}(G, z)|=4 s$, where $s$ is the cardinality of

$$
S=\left\{0 \leq i \leq p:(x y)^{i} z=z(x y)^{i}\right\}
$$

To calculate $s$ observe that

$$
\begin{aligned}
(x y)^{i} \in \mathrm{C}(G, z) & \Longleftrightarrow(x y)^{i} z=z(x y)^{i} \\
& \Longleftrightarrow(x y)^{i} z=(x y)^{-r i} z \\
& \Longleftrightarrow(x y)^{i(r+1)}=1 \\
& \Longleftrightarrow i(r+1) \in(p+1) \mathbb{Z} .
\end{aligned}
$$

Hence, $s$ is the order of the kernel of the group homomorphism

$$
f: \mathrm{Z}_{p+1} \rightarrow \mathrm{Z}_{p+1} ; \ell+(p+1) \mathbb{Z} \mapsto(r+1) \ell+(p+1) \mathbb{Z}
$$

whose image $\langle r+1\rangle$ has order $(p+1) /(r+1, p+1)$. Consequently, $|\operatorname{ker} f|=$ $(r+1, p+1)$, which implies

$$
|\mathrm{C}(G, z)|=4 s=4(r+1, p+1) .
$$

To finish we need the value of $\left|\theta\left(\mathrm{C}\left(\Lambda, c_{3}\right)\right)\right|$. By Theorem 3 in [7] we know that

$$
\mathrm{C}\left(\Lambda, c_{3}\right)=\left\langle c_{3}\right\rangle \oplus\left(\left\langle\left(c_{2} c_{3}\right)\right\rangle *\left\langle\left(c_{3} c_{4}\right)\right\rangle\right)
$$

which implies

$$
\theta\left(\mathrm{C}\left(\Lambda, c_{3}\right)\right)=\langle y, z\rangle \simeq \mathrm{Z}_{2} \oplus \mathrm{Z}_{2} .
$$

Hence, from (4.1) and (4.2) it follows that $\operatorname{Fix}(z)$ has $(r+1, p+1)$ arcs.

Example 4.2. Non-orientable Bujalance-May surfaces. Let $p \geq 2$ with $p \neq 3,6,15$ and let $X=\mathcal{H} / \Gamma$ be a bordered Klein surface of algebraic genus $p$ whose automorphism group $G$ has order $4 p$. Bujalance proved in Theorem 2.5 of [2] that in this situation the surface $X$ is hyperelliptic, the group $G=\operatorname{Aut}(X)=\Lambda / \Gamma$ is the dihedral group

$$
G=\mathrm{D}_{2 p}=\left\langle x, y: x^{2}, y^{2},(x y)^{2 p}\right\rangle
$$

and the signature of $\Lambda$ is $(0,+,[-],\{(2,2,2,2 p)\})$. The corresponding canonical epimorphism $\theta: \Lambda \rightarrow G$ is defined by the assignment

$$
\theta(e)=1, \quad \theta\left(c_{0}\right)=\theta\left(c_{4}\right)=x, \quad \theta\left(c_{1}\right)=1, \quad \theta\left(c_{2}\right)=(x y)^{p}, \quad \theta\left(c_{3}\right)=y .
$$

Using the formulae in Theorems 3.2, 3.3 and 3.4, and taking into account that $(x y)^{p} y$ 
is conjugate to $x$ if $p$ is odd and it is conjugate to $y$ if $p$ is even, we obtain:

\begin{tabular}{|c|c|c|c|c|}
\hline $\begin{array}{c}\text { Representative } \\
\text { of a conjugacy } \\
\text { class }\end{array}$ & $\begin{array}{l}\text { Isolated fixed } \\
\text { points of the } \\
\text { first type }\end{array}$ & $\begin{array}{c}\text { Isolated fixed } \\
\text { points of the } \\
\text { second type }\end{array}$ & Ovals & Arcs \\
\hline$x$ & 0 & $\begin{cases}2 & p \text { odd } \\
0 & p \text { even }\end{cases}$ & 0 & 1 \\
\hline$y$ & 0 & $\begin{cases}0 & p \text { odd } \\
2 & p \text { even }\end{cases}$ & 1 & 0 \\
\hline$(x y)^{p}$ & 0 & 0 & 0 & $p$ \\
\hline$x y$ & 0 & 2 & 0 & 0 \\
\hline
\end{tabular}

Remark 4.3. For the surfaces $X$ in Examples 4.1 and 4.2 , the sets $\operatorname{Fix}(X)$ do not contain fixed points of the first type. Indeed, in order to construct an action $\theta: \Lambda \rightarrow G$ on a surface $X$ such that $\operatorname{Fix}(X)$ has isolated points of both the first and second type, ovals and arcs, the order of the group $G$ must be dramatically reduced. Namely:

Theorem 4.4. Let $G$ be a group acting on a bordered Klein surface $X$ of algebraic genus $p \geq 2$ whose set $\operatorname{Fix}(X)$ of fixed points contains isolated fixed points of both types, ovals and arcs. Then $G$ has at most $2(p-1)$ elements. Furthermore a given group $G$ is the automorphism group of such a surface if and only if it is generated by four involutions $a, b, c, d$ such that $a b$ and bc have order 2 , and the bound $2(p-1)$ for the order of $G$ is attained if and only if $p$ is odd.

Proof. Let us represent $X=\mathcal{H} / \Gamma$, where $\Gamma$ is a surface NEC group, and let $\theta: \Lambda \rightarrow G$ be a smooth epimorphism such that $\operatorname{ker} \theta=\Gamma$. Hence $G \simeq \Lambda / \Gamma$ and, by Theorem 3.2, $\Lambda$ has a proper period, because $G$ contains an automorphism of $X$ with an isolated fixed point of the first type. On the other hand, since $G$ has an involution whose set of fixed points contains some oval, the group $\Lambda$ has, by Theorem 3.3, three consecutive canonical reflections mapped by the epimorphism $\theta$ into involutions. Furthermore, $X$ being a bordered surface, there exists a canonical reflection in $\Lambda$ mapped by $\theta$ into the trivial element. Consequently, the hyperbolic area of $\Lambda$ satisfies $\mu(\Lambda) \geq \pi$ and so, by Riemann-Hurwitz formula, $|G| \leq 2(p-1)$. Even more, this bound is attained if and only if the signature of $\Lambda$ is

$$
(0 ;+;[2] ;\{(2,2,2,2)\})
$$

and, up to a cyclic permutation of the canonical reflections of $\Lambda$, the canonical epimorphism $\theta: \Lambda \rightarrow G$ is induced by the assignment:

$$
\begin{array}{r}
\theta\left(x_{1}\right)=\theta\left(e_{1}\right)=d, \quad \theta\left(c_{0}\right)=a, \quad \theta\left(c_{1}\right)=b, \\
\theta\left(c_{2}\right)=c, \quad \theta\left(c_{3}\right)=1, \quad \theta\left(c_{4}\right)=d a d,
\end{array}
$$

where $a, b, c, d, a b$ and $b c$ are involutions. Notice that since $a, b$ and $a b$ are involutions, the order of $G$ is a multiple of 4 . 
Conversely, given a group $G$ generated by four involutions $a, b, c, d$ such that $a b$ and $b c$ have order 2 , and an NEC group $\Lambda$ whose signature is the one defined in (4.3), the assignment in (4.4) induces a group epimorphism $\theta: \Lambda \rightarrow G$ such that for $\Gamma=\operatorname{ker} \theta$, the quotient $X=\mathcal{H} / \Gamma$ is a bordered Klein surface of algebraic genus $p$ admitting $G$ as a group of automorphisms fixing subsets of all kinds.

Concerning the sharpness of the bound $2(p-1)$ observe that if this is the case, and since $|G|$ is a multiple of 4 , necessarily $p$ is odd. Conversely, suppose that $p$ is odd and let us see that the bound $2(p-1)$ is attained under this assumption. Consider the finite groups with presentation

$$
\begin{aligned}
& G_{1}=\left\langle x, y: x^{2}, y^{2},(x y)^{p-1}\right\rangle=\mathrm{D}_{p-1}, \text { for } p \geq 3, \text { and } \\
& G_{2}=\left\langle x, y, z: x^{2}, y^{2}, z^{2},(x y)^{(p-1) / 2},(x z)^{2}, z y z(x y)^{r} x\right\rangle,
\end{aligned}
$$

where $p \geq 5$ and $r^{2} \equiv 1 \bmod (p-1) / 2$, which is a semidirect product $G_{2}=$ $\mathrm{D}_{(p-1) / 2} \rtimes \mathrm{Z}_{2}$. It is straightforward to check that an NEC group $\Lambda$ whose signature is given in (4.3) and the epimorphism $\theta: \Lambda \rightarrow G=G_{i}$ induced by the assignment (4.4), for $i=1,2$, where $a=c=x, d=y$ and

$$
b=\left\{\begin{array}{cc}
(x y)^{(p-1) / 2} & \text { if } G=G_{1}, \\
z & \text { if } G=G_{2},
\end{array}\right.
$$

do the job.

We finish this section by computing, in the next examples, the number of isolated fixed points of the first and the second type, the number of ovals and the number of arcs, of the set of fixed points of the involutions of the surfaces constructed in Theorem 4.4. We distinguish accordingly the automorphism group of the involved surface is either $G_{1}$ or $G_{2}$. In what follows $\Lambda$ is an NEC group whose signature is defined in (4.3), the epimorphism $\theta: \Lambda \rightarrow G_{i}$ is given by (4.4) and (4.7) and the presentation of $G_{i}$ is provided by (4.5) and (4.6) above. To abbreviate, we denote $q=(p-1) / 2$.

Groups of type 1. To avoid a cumbersome distinction of cases, we assume $p \neq 5$ to guarantee that $(y x)^{2}$ and $(x y)^{q}$ are not conjugate in $G_{1}$. Using the formulae in Theorems 3.2, 3.3 and 3.4, it follows that the number of isolated fixed points of the first and the second type, the number of fixed ovals and the number fixed arcs of the (non-conjugate) involutions of the surface $X=\mathcal{H} / \Gamma$, where $\Gamma=\operatorname{ker} \theta$, are the following:

\begin{tabular}{|c|c|c|c|c|}
\hline $\begin{array}{c}\text { Representative } \\
\text { of a conjugacy } \\
\text { class }\end{array}$ & $\begin{array}{c}\text { Isolated fixed } \\
\text { points of the } \\
\text { first type }\end{array}$ & $\begin{array}{c}\text { Isolated fixed } \\
\text { points of the } \\
\text { second type }\end{array}$ & Ovals & Arcs \\
\hline$x$ & 0 & $\begin{cases}4 & q \text { even } \\
0 & q \text { odd }\end{cases}$ & 1 & 2 \\
$y$ & $\begin{cases}0 & q \text { even } \\
4 & q \text { odd } \\
0\end{cases}$ & 0 & 0 \\
$(x y)^{q}$ & 0 & $q$ & 0 \\
\hline
\end{tabular}


Groups of type 2. Now we calculate the number of isolated fixed points of the first and the second type, the number of fixed ovals and the number of fixed arcs of the (non-conjugate) involutions of the surface $X=\mathcal{H} / \Gamma$, when $G=G_{2}=\mathrm{D}_{q} \rtimes \mathrm{Z}_{2}$ and $\Gamma=\operatorname{ker} \theta$ for the epimorphism $\theta: \Lambda \rightarrow G_{2}$ defined in (4.4) and (4.7) above. We distinguish two subcases according to the parity of $q$.

Subcase 1: For $p \equiv 1 \bmod (4)$, that is, $q$ is even

\begin{tabular}{|c|c|c|c|c|}
\hline $\begin{array}{c}\text { Representative } \\
\text { of a conjugacy } \\
\text { class }\end{array}$ & $\begin{array}{c}\text { Isolated fixed } \\
\text { points of the } \\
\text { first type }\end{array}$ & $\begin{array}{c}\text { Isolated fixed } \\
\text { points of the } \\
\text { second type }\end{array}$ & Ovals & Arcs \\
\hline$x$ & 0 & 0 & 2 & 4 \\
$y$ & 4 & 0 & 0 & 0 \\
$z$ & 0 & $4(r-1, q)$ & 0 & 0 \\
$x z$ & 0 & 0 & $(r+1, q)$ & 0 \\
\hline
\end{tabular}

where, by convention, $(r-1, q)=q$ if $r=1$.

Subcase 2: For $p \equiv 3 \bmod (4)$, that is, $q$ is odd

\begin{tabular}{|c|c|c|c|c|}
\hline $\begin{array}{c}\text { Representative } \\
\text { of a conjugacy } \\
\text { class }\end{array}$ & $\begin{array}{c}\text { Isolated fixed } \\
\text { points of the } \\
\text { first type }\end{array}$ & $\begin{array}{c}\text { Isolated fixed } \\
\text { points of the } \\
\text { second type }\end{array}$ & Ovals & Arcs \\
\hline$y$ & 4 & 0 & 2 & 4 \\
$z$ & 0 & 0 & $(r+1, q)$ & 0 \\
$x z$ & 0 & $4(r-1, q)$ & 0 & 0 \\
\hline
\end{tabular}

Acknowledgements. The authors are grateful to the anonymous referee for pointing out many interesting remarks and comments that contributed significatively to clarify both the general exposition and some particular results in the article.

\section{References}

[1] Alling, N. L. And Greenleaf, N.: Foundations of the theory of Klein surfaces. Lecture Notes in Mathematics 219, Springer-Verlag, Berlin-New York, 1971.

[2] Bujalance, E.: On compact Klein surfaces with a special automorphism group. Ann. Acad. Sci. Fenn. 22 (1997), no. 1, 15-20.

[3] Bujalance, E., Cirre, J. F., Gamboa, J. M. and Gromadzki, G.: Symmetries of Riemann surfaces. Lecture Notes in Mathematics 2007, Springer-Verlag, BerlinHeidelberg, 2010.

[4] Bujalance, E., Costa, A. F., Natanzon, S. M. and Singerman, D.: Involutions of compact Klein surfaces. Math. Z. 211 (1992), no. 3, 461-478. 
[5] Bujalance, E., Etayo, J. J., Gamboa, J. M. and Gromadzki, G.: Automorphism groups of compact bordered Klein surfaces. A combinatorial approach. Lecture Notes in Mathematics 1439, Springer-Verlag, Berlin, 1990.

[6] Gromadzki, G.: On a Harnack-Natanzon theorem for the family of real forms of Riemann surfaces. Journal Pure Appl. Algebra 121 (1997), no. 3, 253-269.

[7] Gromadzki, G.: Symmetries of Riemann surfaces from a combinatorial point of view. In Topics on Riemann surfaces and Fuchsian groups (Madrid, 1998), 91-112. London Math. Soc. Lecture Note Series 287, Cambridge University Press, Cambridge, 2001.

[8] Gromadzki, G.: On fixed points of automorphisms of non-orientable unbordered Klein surfaces. Publ. Mat. 53 (2009), no. 1, 73-82.

[9] Izquierdo, M. And Singerman, D.: On the fixed-point set of automorphisms of non-orientable surfaces without boundary. In The Epstein birthday schrift, 295-301. Geom. Topol. Monographs 1, Geom. Topol. Publ., Coventry, 1998.

[10] Macbeath, A. M.: The classification of non-euclidean plane crystallographic groups. Canad. J. Math. 19 (1967), 1192-1205.

[11] Macbeath, A. M.: Action of automorphisms of a compact Riemann surface on the first homology group. Bull. London Math. Soc. 5 (1973), 103-108.

[12] MAY, C. L.: A bound for the number of automorphisms of a compact Klein surface with boundary. Proc. Amer. Math. Soc. 63 (1977), no. 2, 273-280.

[13] Singerman, D.: Non-Euclidean crystallographic groups and Riemann surfaces. Ph. D. Thesis, Univ. of Birmingham, 1969.

[14] Singerman, D.: On the structure of non-Euclidean crystallographic groups. Proc. Cambridge Philos. Soc. 76 (1974), 233-240.

[15] Wilkie, H. C.: On non-euclidean crystallographic groups. Math. Z. 91 (1966), 87-102.

Received February 3, 2010; revised July 13, 2010.

José Manuel Gamboa: Departamento de Álgebra, Facultad de Matemáticas, Universidad Complutense. 28040-Madrid, Spain.

E-mail: jmgamboa@mat.ucm.es

Grzegorz Gromadzki: Institute of Mathematics, University of Gdańsk, Wita Stwosza 57, 80-952 Gdańsk, Poland.

E-mail: greggrom@math.univ.gda.pl

The first author is supported by Spanish GAAR MTM2008-00272, Proyecto Santander Complutense PR34/07-15813, GAAR Grupos UCM 910444 and by Geometría real MTM2011-22435. The second author is supported by the Research Grant NN 201366436 of the Polish Ministry of Sciences and Higher Education. 